\title{
A dinâmica do aleitamento materno entre famílias em vulnerabilidade social: o que revela o sistema de busca ativa
}

\author{
Breastfeeding dynamics among families \\ on social vulnerability: findings \\ of active search system
}

Semíramis Martins Álvares DOMENE ${ }^{1}$

Maria Angélica Tavares de MEDEIROS ${ }^{1}$

Paula Andrea MARTINS ${ }^{1}$

RE S U M O

\section{Objetivo}

Descrever as práticas de amamentação em grupos vulneráveis residentes na área de cobertura do Distrito Noroeste de Campinas.

\section{Métodos}

Foi realizado um estudo transversal, utilizando levantamento de caráter censitário, denominado busca ativa, em visitas a todos os domicílios da área estudada. Foi feito um estudo exploratório das práticas de aleitamento materno em população moradora de região urbana periférica em vulnerabilidade social, com aproximadamente 165 mil habitantes, atendidos por três unidades básicas de saúde.

\section{Resultados}

Foram encontradas 1139 crianças com até seis anos de idade, com 72,6\% das famílias abaixo da linha de pobreza. A amamentação ocorreu em 93,3\% dos casos, com mediana de aleitamento materno de 8 meses (IC 95\% 7,0 - 8,9 meses), tempo de aleitamento materno exclusivo com mediana de 4 meses (IC 95\% 3,9 - 4,1 meses), sendo que mais de $20 \%$ das crianças recebiam outros alimentos já no primeiro mês. Grande parte das famílias referiu não ter recebido orientação sobre o aleitamento materno nas unidades básicas de saúde.

\section{Conclusão}

O tempo de aleitamento materno e de aleitamento materno exclusivo na área do estudo, encontrado por busca ativa, foi superior à média nacional e também ao observado em populações com características socioeconômicas semelhantes, o que reforça a importância da incorporação dessa estratégia pela Atenção Básica, nas ações de promoção do aleitamento materno.

Termos de indexação: Aleitamento materno. Lactente. Serviços de saúde.

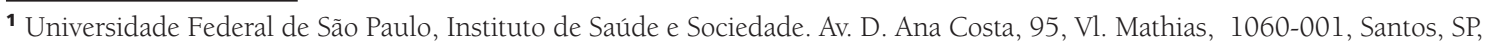
Brasil. Correspondência para/Correspondece to: S.M.A. DOMENE. E-mail: <semiramis.domene@unifesp.br>.
} 


\section{A B S T R A C T}

\section{Objective}

This study describes the breastfeeding practices of vulnerable groups living in an area covered by the Northwest Health District of Campinas (SP), Brazil.

\section{Methods}

A cross-sectional study was done by visiting all families in the studied area and performing a census-like survey called active search. An exploratory study was done of the breastfeeding practices of a socially vulnerable population living in the outskirts of Campinas. This area has approximately 165,000 inhabitants and three primary healthcare facilities.

\section{Results}

The survey found 1,139 children under six years of age and $72.6 \%$ of the families were below the poverty line; 93.3\% of the children were breastfeed for a median of 8 months (CI 95\% 7.0 - 8.9 months); exclusive breastfeeding lasts a median of 4 months (CI 95\% 3.9 - 4.1 months), and more than 20\% of the children were given other foods during their first month of life. Most of the families reported that the primary healthcare facilities gave them no information on breastfeeding.

\section{Conclusion}

Breastfeeding and exclusive breastfeeding in the studied area found by active search lasted longer than the national average and longer than those of families with similar socioeconomic characteristics. This reinforces the importance of primary healthcare incorporating this strategy in actions that promote breastfeeding.

Indexing terms: Breast feeding. Infant. Health services.

\section{N T R O D U Ç Ã O}

A semelhança do perfil de agravos à saúde observada entre grupamentos de diferentes estratos socioeconômicos revela que o crescimento do sobrepeso e das doenças crônicas exige abordagens diferenciadas em cada situação. De forma aparentemente paradoxal, apesar da estabilização econômica que ocorreu no Brasil a partir dos anos 90, com ampliação do acesso a alimentos, a insegurança por carência alimentar era presente, em 2004, em 34,5\% dos domicílios do País.

O incentivo ao aleitamento materno está entre as ações promotoras de segurança alimentar, na medida em que as recomendações nacionais e internacionais preconizam o estímulo a essa prática, sem complementação com nenhum outro alimento até o sexto mês de vida ${ }^{2,3}$. No Brasil, a prevalência de Aleitamento Materno (AM) ao sexto mês aumentou de $22 \%$ para $69 \%$ entre os anos de 1975 e 19994, passando de 296 dias, em 1999, para 342 dias, em 2008, nas capitais e no Distrito Federal. A duração do Aleitamento Materno Exclusivo (AME), de 24 dias em 1999, passou a ser de 54 dias em 2008 - ou seja, mais que dobrou em 10 anos $^{5}$. Os determinantes da interrupção precoce do aleitamento materno, ou de práticas alimentares que promovem o desmame, foram amplamente estudados, incluindo crenças e tabus, necessidade da mãe que amamenta em retornar ao trabalho ${ }^{6-8}$ e até mesmo o despreparo das equipes de saúde ${ }^{9}$.

Estudos de base populacional sobre a utilização dos serviços de saúde mostram que a Estratégia de Saúde da Família (ESF) caminha para diminuir a iniquidade da assistência, especialmente no nível da atenção básica ${ }^{10,11}$. De outro lado, o acesso ao serviço público tem se estendido a uma parcela crescente de indivíduos que também usam serviços conveniados ou particulares ${ }^{12}$. Apesar disso, ainda é baixa a cobertura das ações dirigidas a grupos vulneráveis a cargo das Unidades Básicas de Saúde (UBS) ${ }^{11,13}$.

A busca ativa, como ferramenta específica da vigilância epidemiológica ${ }^{14,15}$, a partir da qual as equipes de saúde se deslocam até o território com o propósito de detectar agravos e intervir sobre eles, é um modo de ampliar o alcance das 
ações de saúde. Este estudo pretendeu descrever as práticas de amamentação em grupos vulneráveis, por meio de levantamento de dados obtidos em busca ativa, entre os moradores da área de cobertura do Distrito Noroeste de Campinas.

\section{MÉ TO D O S}

Trata-se de estudo transversal, desenvolvido no Distrito Noroeste de Campinas, região que contava, segundo o censo de 2000, com 15,2\% de pessoas residentes em aglomerados subnormais ${ }^{(2)}$, acima da média do município, de 13,2\% ${ }^{16}$. Com população estimada em 165.497 habitantes para o ano de $2005^{17}$, calculou-se que uma amostra de 599 crianças com até seis anos seria suficiente para obter um poder de 99\% (1- $\beta$ ) com um nível de significância de 0,05 (1- $\alpha)$.

O Distrito Noroeste possui 12 UBS, tendo sido selecionadas 3: Integração, Jardim Ipaussurama e Pedro Aquino, as quais apresentam território definido, atendendo a média de 20 mil habitantes cada uma, com 3 a 4 equipes de saúde da família por unidade ${ }^{16}$. Esse é também o território de parceria universidade/serviço, no desenvolvimento de atividades de ensino, pesquisa e extensão da Pontifícia Universidade Católica de Campinas.

Como critérios de inclusão foram consideradas a idade de até 6 anos e a residência na área de cobertura das UBS Integração, Jardim Ipaussurama e Pedro Aquino. Foram excluídas do estudo as crianças portadoras de qualquer afecção aguda ou crônica que afetasse o seu estado nutricional.

As equipes de pesquisa, formadas por nutricionistas capacitados, juntamente com os agentes de saúde de cada UBS, percorreram, no ano de 2007, as ruas das áreas de abrangência, verificando, entre todos os domicílios, aqueles que eram elegíveis, ou seja, em que residissem crianças com até seis anos de idade, para entrevista com a mãe ou um responsável. As famílias que ainda não constavam no cadastro das UBS foram tam- bém visitadas, a fim de verificar a presença de crianças menores de seis anos.

Foi preenchido um questionário com as respostas obtidas nas entrevistas com os responsáveis pelas crianças, no qual foram coletados dados sobre renda e escolaridade dos mesmos, condições de habitação e saneamento do domicílio, dados sobre aleitamento materno, uso de fórmulas infantis, história recente de infecções, parasitoses e uso de suplementos nutricionais.

Foram realizadas análises estatísticas descritivas. Para avaliar a frequência de aleitamento materno em menores de dois anos, foi utilizada análise de sobrevida, que permitiu estimar a mediana de aleitamento materno total e exclusivo, bem como seus intervalos de confiança (95\%).

O protocolo do estudo foi aprovado pelo Comitê de Ética para Pesquisa com Seres Humanos da Pontifícia Universidade Católica Campinas (PUC-Campinas), processo SISNEP $n^{\circ}$ 1067.0.147.000-06, sendo os dados coletados mediante assinatura do Termo de Consentimento Livre e Esclarecido pelo responsável legal pela criança.

\section{RES U L T A D OS}

Foram encontradas 1139 crianças que atendiam ao critério de inclusão, sendo 568 meninas $(49,9 \%)$ e 571 meninos $(51,1 \%)$, das quais $509(44,7 \%)$ frequentavam creches.

As entrevistas foram realizadas com as mães das crianças $(84,8 \%)$, seguidas das avós $(5,7 \%)$, dos pais $(3,9 \%)$, do tio ou tia $(3,1 \%)$, ou de respondente com outro grau de parentesco. Quanto à escolaridade do responsável pela criança, 41,0\% dos entrevistados tinham até o primeiro grau. Considerando o número médio de habitantes no domicílio (5, Desvio-Padrão - $\mathrm{DP}=2$, com mediana de 4; mínimo 2 e máximo 15) e a renda familiar total, aproximadamente $72,6 \%$ das famílias estão abaixo da linha de pobreza de 0,5

2 Favelas, núcleos residenciais, ocupações e ocupações em fase de regularização. 
salário-mínimo familiar per capita. Os indicadores socioeconômicos estão expostos na Tabela 1.

Em relação à proporção de aleitamento materno, 93,3\% das mães amamentaram seus filhos, com mediana de AM de 8 meses (IC 95\% 7,0 - 8,9 meses). Poucas crianças ainda estavam

Tabela 1. Características socioeconômicas das famílias, idade e características de nascimento das crianças com até seis anos. Distrito Noroeste de Campinas (SP), 2007.

\begin{tabular}{|c|c|c|}
\hline Característica & $\mathrm{n}$ & $\%$ \\
\hline \multicolumn{3}{|c|}{ Escolaridade do responsável ( $n=1$ 133) } \\
\hline nenhuma & 21 & 1,9 \\
\hline 1 a $4^{a}$ série & 209 & 18,4 \\
\hline 5 a $8^{\mathrm{a}}$ série & 464 & 41,0 \\
\hline $2^{\circ} \mathrm{grau}$ & 406 & 35,8 \\
\hline $3^{\circ}$ grau & 33 & 2,9 \\
\hline \multicolumn{3}{|c|}{ Renda familiar mensal (salários-mínimos) ( $n=1$ 095) } \\
\hline$<1$ & 241 & 22,0 \\
\hline 1 a 2 & 554 & 50,6 \\
\hline 3 ou mais & 300 & 27,4 \\
\hline \multicolumn{3}{|c|}{ Destino do esgoto domiciliar ( $n=1$ 029) } \\
\hline Céu aberto & 166 & 14,7 \\
\hline Rio/córrego & 195 & 17,3 \\
\hline Fossa & 137 & 12,1 \\
\hline Rede pública & 631 & 55,9 \\
\hline \multicolumn{3}{|c|}{ Tratamento da água domiciliar ( $n=1$ 029) } \\
\hline nenhum & 421 & 37,3 \\
\hline Fervura & 29 & 2,6 \\
\hline Filtro & 451 & 39,9 \\
\hline Mineral & 224 & 19,8 \\
\hline Cloração & 4 & 0,4 \\
\hline \multicolumn{3}{|l|}{ Idade ( $n=1$ 139) } \\
\hline$<2$ anos & 353 & 31,0 \\
\hline $2 \mid-4$ anos & 309 & 27,1 \\
\hline$\geq 4$ anos & 477 & 41,9 \\
\hline \multicolumn{3}{|c|}{ Peso ao nascer $(n=1$ 019) } \\
\hline$<1500 \mathrm{~g}$ & 12 & 1,2 \\
\hline $15001-2500 \mathrm{~g}$ & 110 & 10,8 \\
\hline $25001-3000 \mathrm{~g}$ & 274 & 26,9 \\
\hline$\geq 3000 \mathrm{~g}$ & 623 & 61,1 \\
\hline \multicolumn{3}{|c|}{ Comprimento ao nascer $(n=944)$} \\
\hline$<45$ & 155 & 16,4 \\
\hline $45 \mid-47$ & 216 & 22,9 \\
\hline $47 \mid-49$ & 330 & 35,0 \\
\hline $49 \mid-51$ & 193 & 20,4 \\
\hline$\geq 51$ & 50 & 5,3 \\
\hline \multicolumn{3}{|c|}{ Período gestacional $(n=974)$} \\
\hline$\leq 37$ semanas & 195 & 20,0 \\
\hline$>37$ semanas & 779 & 80,0 \\
\hline
\end{tabular}

sendo amamentadas ao completarem um ano de vida e um número ainda menor aos dois anos $(14,0 \%)$ (Figura 1). Nenhuma criança foi amamentada por mais de três anos.

A mediana do tempo de aleitamento materno exclusivo foi de 4 meses (intervalo de confiança - IC 95\% 3,9 - 4,1 meses). Mais de $20 \%$ das crianças recebiam outros alimentos já no primeiro mês, e ao sexto mês apenas 10\% das crianças estavam em AME, como se pode ver na Figura 2. Um percentual de $4 \%$ das crianças recebeu aleitamento materno exclusivo até 8 meses ou mais.

Ao considerar a idade das crianças no momento da avaliação, pôde-se observar que mais da metade das menores de quatro meses recebiam leite de vaca não modificado. Quase a totalidade das crianças com menos de dois anos recebia leite de vaca (Tabela 2 ).

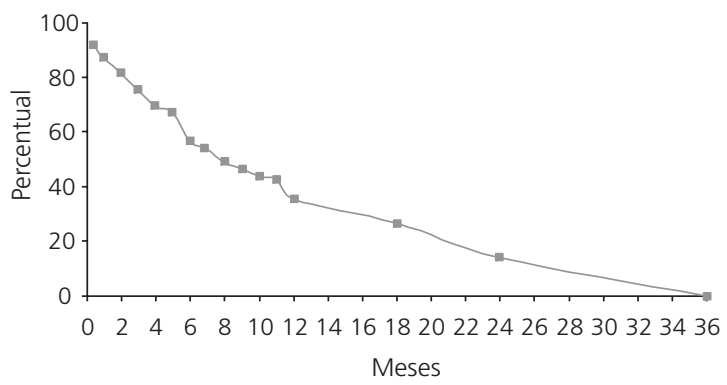

Figura 1. Frequência de aleitamento materno estimado pelo sistema de busca ativa no Distrito Noroeste de Campinas (SP), 2007.

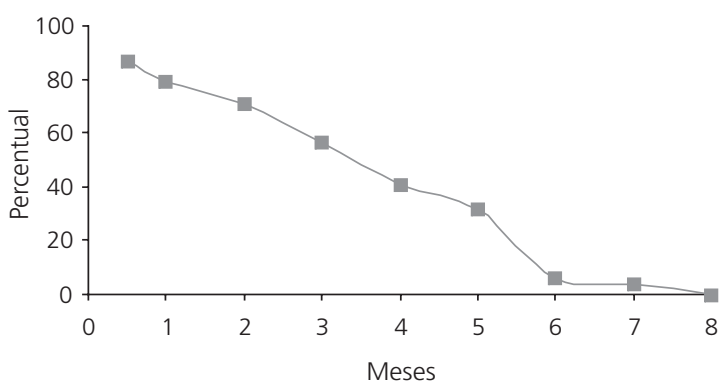

Figura 2. Frequência de aleitamento materno exclusivo estimado pelo sistema de busca ativa no Distrito Noroeste de Campinas (SP), 2007. 
Tabela 2. Utilização de leite de vaca nos dois primeiros anos de vida de crianças com até seis anos. Distrito Noroeste de Campinas (SP), 2007.

\begin{tabular}{lcc}
\hline Padrão & $n$ & $\%$ \\
\hline 4 meses & 35 & 54 \\
6 meses & 63 & 62 \\
12 meses & 147 & 79 \\
24 meses & 314 & 89 \\
\hline
\end{tabular}

Tabela 3. Uso de fórmulas lácteas modificadas e complementos alimentares na dieta de crianças com até seis anos. Distrito Noroeste de Campinas (SP), 2007.

\begin{tabular}{lcc}
\hline Fórmula & $\mathrm{n}$ & $\%$ \\
\hline Leite modificado & 249 & 48,7 \\
Leite modificado e mucilagens & 49 & 9,6 \\
Farinha láctea & 32 & 6,3 \\
Mais do que uma mucilagem & 6 & 1,2 \\
Outros leites modificados & 5 & 1,0 \\
Outros complementos lácteos & 3 & 0,6 \\
\hline Total & 511 & 100,0 \\
\hline
\end{tabular}

Fórmulas lácteas modificadas e complementos alimentares adicionados ao leite estavam presentes no dia alimentar de $45 \%(n=511)$ do total de crianças avaliadas. Na Tabela 3 são informadas as fórmulas e os complementos referidos, bem como suas combinações.

\section{I S C U S S Ã O}

A manutenção de dados atualizados sobre a prevalência de práticas associadas ao aleitamento materno, especialmente o tempo total e o de aleitamento exclusivo, é relevante para monitorar a efetividade da assistência no nível básico da atenção à saúde.

Ações educativas dirigidas à equipe de saúde já mostraram resultados: após intervenção com o protocolo empregado pela Iniciativa Hospital Amigo da Criança ${ }^{18}$, foi observado aumento no tempo de aleitamento exclusivo em município de Minas Gerais, com elevação da duração mediana de 104 para 125 dias, muito embora os autores não tenham constatado alteração na duração total do aleitamento.
Neste estudo, a duração mediana do AME foi de quatro meses, superior à estimada para o País, de 54 dias; no entanto, aos seis meses de idade, apenas $56,3 \%$ das crianças ainda eram amamentadas. Estudo anterior, desenvolvido na mesma região em 2001, durante campanha de vacinação ${ }^{19}$, revelou que $58,2 \%$ das mães referiram não ter recebido orientação sobre a amamentação durante o pré-natal. Nesse mesmo ano, a mediana de AME na região era de 68 dias e a de AM, de 6,4 meses $^{20}$. Tempos de AME e AM como estes, portanto, não são comuns em estudos nacionais; Simon et al..$^{21}$ encontraram mediana de 4 meses para AME e 7 meses para AM entre crianças de bom poder aquisitivo.

Dados obtidos por meio de censo, como os deste estudo, incluem crianças que não chegam ao Centro de Saúde. Tendo em vista que os indicadores de aleitamento encontrados são mais promissores do que os dados nacionais ou os obtidos na mesma região anteriormente, permitindo identificar tendência de melhora, postula-se a que determinantes este aumento deve responder.

Entre os aspectos que comprometem o sucesso da estratégia de saúde da família, está o uso precoce do leite de vaca, oferecido a $54 \%$ das crianças já aos quatro meses de idade. Esse resultado é preocupante, na medida em que, conforme consenso, é inadequada a oferta de leites não modificados antes dos dois anos de vida, em função do risco potencial de alergias e irritações na mucosa intestinal.

Chama atenção o fato de um número grande de crianças ter usado uma fórmula de leite modificado de alto custo, considerando-se as características socioeconômicas da população. Embora seja o alimento ideal na impossibilidade do leite materno, fica evidente a necessidade de estratégias eficientes de promoção do aleitamento materno.

Mesmo após a implantação da ESF, observa-se baixa adesão ao pré-natal (31\% das gestantes) e à puericultura $(20 \% \text { das crianças })^{11}$. A proposição, por parte do Ministério da Saúde, da 
Iniciativa Unidade Básica Amiga da Amamentação (IUBAAM) visa mobilizar as equipes em torno do cuidado primário, tendo por referencial a adoção dos "Dez passos para o sucesso da amamentação", semelhantemente ao que se propôs com o Hospital Amigo da Criança. A qualificação da atenção básica para o enfrentamento das principais variáveis envolvidas na interrupção precoce do aleitamento materno é uma das metas dessas ações.

Um dos aspectos associados à prática do AM parece ser a experiência bem sucedida da geração anterior; crianças que foram amamentadas serão mães que amamentam por tempo maior do que as que não receberam leite materno 22,23 . Diante do fato de que grande parte das famílias entrevistadas não recebeu orientação na UBS, as ações previstas na Estratégia Saúde da Família devem considerar, como mostram os dados levantados por meio do sistema da busca ativa, que o AME conduzido espontaneamente é bem sucedido, conforme os tempos encontrados neste estudo. Contudo, o possível viés de memória entre os entrevistados, uma vez que o levantamento incluiu crianças com até seis anos de idade, deve ser considerado como limitação deste estudo.

\section{CONCLUS Ã O}

O tempo de aleitamento materno e de aleitamento materno exclusivo na área de cobertura do Distrito Noroeste de Campinas, encontrado pelo sistema de busca ativa, foi superior à média nacional e ao observado em populações com características socioeconômicas semelhantes, a partir de dados obtidos nos serviços de saúde, e mesmo a partir de levantamentos anteriores na mesma população. A Estratégia Saúde da Família deve considerar, a partir desta constatação, que as ações de atenção básica serão bem sucedidas na promoção do aleitamento materno se compreenderem que as dinâmicas envolvidas com esta prática respondem a um complexo conjunto de determinantes, muitas vezes de difícil percepção pela equipe. Pequenas interferências, se negativas, podem contribuir para abreviar práticas como esta, cujo estímulo é inquestionavelmente um compromisso do serviço.

\section{A GRADECIMENTO}

Ao Conselho Nacional de Desenvolvimento Científico e Tecnológico (CNPq), pelo financiamento ao projeto do qual se originou este estudo (Edital MCT-CNPq/MS-DAB/SAS - N 51/2005).

\section{COLABORADORES}

S.M.A. DOMENE, M.A.T. MEDEIROS e P.A. MARTINS participaram da concepção, coleta, análise dos dados e redação do manuscrito.

\section{REFERÊ NCIAS}

1. Instituto Brasileiro de Geografia e Estatística. Pesquisa Nacional por Amostra de Domicílios. Segurança alimentar: 2004. Rio de Janeiro; IBGE; 2006.

2. World Health Organization. Global strategy for infant and young child feeding. Procceedings of 54 ${ }^{\text {th }}$ World Health Assembly; 2001 Apr 9; Geneva: WHO; 2001.

3. Brasil. Ministério da Saúde. Secretaria de Atenção à Saúde. Saúde da criança: nutrição infantil: aleitamento materno e alimentação complementar. Brasília: Ministério da Saúde; 2009.

4. Lamounier JA, Bouzada MCF, Janneu AMS, Maranhão AGK, Araújo MFM, Vieira GO, et al. Iniciativa hospital amigo da criança, mais de uma década no Brasil: repensando o futuro. Rev Paul Pediatr. 2008; 26(2):161-9.

5. Brasil. Ministério da Saúde. Tempo médio de aleitamento materno aumenta de 296 para 342 dias em nove anos [acesso 2009 out. 3]. Disponível em: $<$ http://portal.saude.gov.br/portal/>.

6. Venâncio SI, Escudero MML, Kitoko P, Rea MF, Monteiro CA. Freqüência e determinantes do aleitamento materno em municípios do Estado de São Paulo. Rev Saúde Pública. 2002; 36(3):313-8.

7. Vieira GO, Almeida JAG, Silva LR, Cabral VA, Santana PV. Fatores associados ao aleitamento materno e desmame em Feira de Santana, Bahia. Rev Bras Saúde Mater Infant. 2004; 4(2):143-50. 
8. Ramos CV, Almeida JAG. Alegações maternas para o desmame: estudo qualitativo. J Pediatr. 2003; 79(5):385-90.

9. Toma TS, Monteiro CA. Avaliação da promoção do aleitamento materno nas maternidades públicas e privadas do Município de São Paulo. Rev Saúde Pública. 2001; 35(5):409-14.

10. Goldbaum M, Gianini RJ, Novaes HMD, Cesar CLG. Utilização de serviços de saúde em áreas cobertas pelo programa saúde da família (Qualis) no Município de São Paulo. Rev Saúde Pública. 2005; 39(1): 90-9.

11. Fernandes LCL, Bertoldi AD, Barros AJD. Utilização dos serviços de saúde pela população coberta pela estratégia de saúde da família. Rev Saúde Pública. 2009; 43(4):595-603.

12. Dias-da-Costa JS, Gigante DP, Horta BL, Barros FC, Victora CG. Utilização de serviços de saúde por adultos da coorte de nascimentos de 1982 a 2004-5, Pelotas, RS. Rev Saúde Pública. 2009; 42(Supl. 2):51-9.

13. Nascimento ER, Paiva MS, Rodrigues QP. Avaliação da cobertura e indicadores do Programa de Humanização do Pré-natal e Nascimento no município de Salvador, Bahia, Brasil. Rev Bras Saude Mater Infant. 2007; 7(2):191-7.

14. Solymos GMB, Sawaya AL, Ferrari AA, Vieira MFA, Ugnebu $\mathrm{CH}$. Centro de recuperação e educação nutricional: uma proposta para o combate à desnutrição energético-protéica. In: Sawaya, AL, organizador. Desnutrição urbana no Brasil: em um período de transição. São Paulo: Cortez; 1997. p.161-93.

15. Souza MHN, Ferrari AA. Prevenção da desnutrição energético-protéica na comunidade. In: Sawaya AL, organizador. Desnutrição urbana no Brasil: em um período de transição. São Paulo: Cortez; 1997. p.85-97.
16. Campinas. Secretaria Municipal de Saúde. Saúde em números 2004. [acesso 2007 fev. 15]. Disponível em: <http://www.campinas.sp.gov.br/saude/ dados/populac/censo2000_id_detalh_norte_pdf. htm>.

17. Instituto Brasileiro de Geografia e Estatística. Resultados do universo censo 2000. [acesso 2004 jan. 19]. Disponível em: <http.www.ibge.gov.br/ ibgecidades@>.

18. Caldeira AP, Aguiar GN, Magalhães WAC, Fagundes GC. Conhecimentos e práticas de promoção do aleitamento materno em equipes de saúde da família em Montes Claros, Minas Gerais, Brasil. Cad Saúde Pública. 2007; 23(8):1965-70.

19. Volpini CCA, Moura EC. Determinantes do desmame precoce no distrito noroeste de Campinas. Rev Nutr. 2005; 18(3):311-9. doi: 10.1590/\$1514-5273 2005000300003.

20. Camilo DF, Carvalho RVB, Oliveira EF, Moura EC. Prevalência da amamentação em crianças menores de dois anos vacinadas nos centros de saúde escola. Rev Nutr. 2004; 17(1):29-36. doi: 10.1590/S1514-5 2732004000100003.

21. Simon VGN, Souza JMP, Souza SB. Aleitamento materno, alimentação complementar, sobrepeso e obesidade em pré-escolares. Rev Saúde Pública. 2009; 43(1):60-9.

22. Horta BL, Victora CG, Gigante DP, Santos J, Barros FC. Duração da amamentação em duas gerações. Rev Saúde Pública. 2007; 41(1):13-8.

23. Araujo RMA, Almeida JAG. Aleitamento materno: o desafio de compreender a vivência. Rev Nutr. 2007; 20(4):431-8. doi: 10.1590/\$1415-5273200 7000400010 .

Recebido em: 11/1/2010

Versão final reapresentada em: 13/8/2010

Aprovado em: 23/9/2010 
